\title{
Unmoderated Posters Evidence-Based Medicine and Outcomes
}

\author{
UP-52 \\ Clavien Classification in Urology: Is There Concordance Among \\ Post-graduate Trainees and Attending Urologists? \\ Elkoushy, Mohamed; Luz, Murilo; Benidir, Tarik; Aldousari, Saad; Aprikian, \\ Armen; Andonian, Sero \\ McGill University Health Centre, Montreal, QC, Canada \\ Purpose: One of the limitations of the Revised Clavien-Dindo Classification \\ System (RCCS) is that subjective interpretation of postoperative compli- \\ cations leads to variation in grade assignment. Since Quality Assurance \\ case presentations are usually prepared by Post-Graduate Trainees (PGTs) \\ in academic centres, the aim of the present study was to compare the \\ agreement rate between urology PGTs and attending urologists applying \\ the RCCS.
}

Methods: Twenty postoperative complications were selected from urology service Quality Assurance meeting minutes spanning one year at a tertiary care centre. The cases were from both adult and pediatric sites and included both minor and major complications. After a briefing session to review the RCCS, the survey was administered to 16 attending urologists and 16 PGTs. Concordance rates between the two groups were calculated for each case and for the whole survey. Inter-rater agreement was calculated by kappa statistics.

Results: There was good overall agreement rate of $81 \%(30-100 \%)$ when both groups were compared. Thirteen of the 20 cases $(65 \%)$ held an agreement rate above $80 \%(\mathrm{k}=0.753, p<0.001)$ including $3(15 \%)$ cases with $100 \%$ agreement. There were only two cases where the scores given by PGTs were significantly different from that given by attending urologists $(p \leq 0.03)$. However, in these 3 cases, there was no pattern of one group grading worse than the other. There was no significant difference between both groups in terms of overall RCCS grades $(p=0.12)$. When all participants were compared as one group, there was good overall interrater agreement rate of $75 \%(\mathrm{k}=0.71)$. Although agreement among PGTs was higher than among attending urologists $[82 \%(\mathrm{k}=0.79)$ vs. $69 \%[\mathrm{k}$ $=0.64)]$, this was not significant $(p=0.68)$.

Conclusions: There was good overall agreement among PGTs and attending urologists in application of the RCCS in urology. Therefore, it is appropriate for PGTs to complete the Quality Assurance meeting reports.

\section{UP-53}

Participation Bias in a Prostate Cancer Quality of Life Survey

Kim, Brian ${ }^{1}$; Porter, Kimberly²; Contreras, Richard ${ }^{2}$; Salazar, Luis ${ }^{1}$; Thomas, Anil $^{1}$; Slezak, Jeffrey ${ }^{2}$; Jacobsen, Steven ${ }^{2}$; Chien, Gary ${ }^{1}$

${ }^{1}$ Kaiser Permanente, Los Angeles, CA, United States; ${ }^{2}$ Kaiser Permanente, Pasadena, CA, United States

Introduction and Objectives: Patient-reported surveys are an invaluable clinical tool for measuring the impact of a disease or response to a treatment. Surveys are often marred, however, by poor participation rates potentially introducing bias if participants differ from non-participants. The study objective was to identify potential demographic and clinical factors associated with participation in a prostate cancer quality of life survey. Methods: From March 2011 to September 2012, all men with biopsyproven prostate cancer were asked to participate in a quality of life study at 12 regional hospitals at Kaiser Permanente, Southern California. Men completed the Expanded Prostate Cancer Index Composite (EPIC-26) at the time of their prostate biopsy (baseline) and at specific follow-up intervals after treatment $(1,3,6,12,18$, and 24 months). Surveys were administered in either English or Spanish. Select demographic and clinical parameters were compared between participants (those that completed a baseline and any follow-up survey) and non-participants (those that completed only a baseline survey) using chi-squared and ANOVA tests. Results: A total of 1698 men were enrolled (1073 participants; 625 nonparticipants; participation rate $63.2 \%$ ). Men who participated tended to be older, partnered, primarily English-speaking, Caucasian, and living in regions with higher education and income levels $(p<0.05)$. Men with a positive family history for prostate cancer were also more likely to continue in the study $(p=0.03)$. PSA levels, biopsy Gleason sum, Charlson comorbidity index, BMI, and smoking status were similar amongst study participants and non-participants.

Conclusions: These data suggest that the survey results will have to be interpreted in light of these demographic differences, as they may be associated with quality of life. Moreover, this information can be used for targeted retention efforts in this and other prostate cancer quality of life studies.

\section{UP-54}

A Systematic Review of Direct Cost of Initial Treatments for Localized Prostate Cancer

Sanyal, Chiranjeev; Aprikian, Armen; Chevalier, Simone; Dragomir, Alice McGill University, Montreal, QC, Canada

Introduction and Objectives: Prostate cancer $(\mathrm{PCa})$ is the most common cancer among Canadian men. The growing healthcare expenditure to treat localized PCa has created greater need for the appraisal of direct costs of initial treatments for localized PCa. This will assist in healthcare planning and policy by identifying the economic burden of initial treatments for localized PCa. The objectives of this study were to systematically review and synthesize the literature on direct costs of initial treatments for localized PCa and to review the methodological considerations.

Methods: A systematic search of peer-reviewed articles on direct costs of initial treatments for localized PCa was conducted on bibliographic databases. Studies were appraised for methodological considerations and mean direct costs by initial treatments for localized PCa. Direct costs reported by the studies were standardized to 2011 Canadian dollars (CAD).

Results: Eighteen articles were included in the review. The mean direct costs in 2011 CAD varied considerably and ranged from: $\$ 28,218$ to $\$ 65,946$ for intensity modulated radiotherapy (IMRT)/three dimensional conformal radiotherapy; $\$ 8053$ to $\$ 32,655$ for robot-assisted radical prostatectomy (RARP); $\$ 1031$ to $\$ 43,593$ for external beam radiation therapy (EBRT); $\$ 528$ to $\$ 38,189$ for prostatectomy; and $\$ 972$ to $\$ 8794$ for active surveillance (AS). Our study shows a trend of increasing costs with newer treatments (i.e., IMRT, RARP) compared to conventional treatments (i.e. prostatectomy, EBRT) or AS.

Conclusions: The existing literature lacks cost analysis on initial treatments for localized PCa specific to publicly funded healthcare systems. Healthcare resource use and unit costs are sensitive to variations across healthcare systems that limit generalizability and transferability of cost estimates. Hence, country specific costs are essential for decision makers and healthcare planners to efficiently allocate competing healthcare resources. 


\section{UP-55}

Real Time Active Surveillance for Localized Prostate Cancer: A Dual Surgeon Experience

Patel, Premal ${ }^{1}$; Reikie, Brian'; Carlson, Kevin²; Baverstock, Richard ${ }^{2}$

${ }^{1}$ Faculty of Medicine, University of Calgary, Calgary, AB, Canada; ${ }^{2}$ vesia [Alberta Bladder Centre], Calgary, AB, Canada

Introduction and Objective: Active surveillance has become the standard of care for individuals with favourable-risk prostate cancer. This entails expectant management rather than immediate therapy. However, this fails to provide a "real life" example as there are individuals who avoid biopsies or wish to stay on active surveillance with unfavourable disease. We compared the timing of prostate cancer surveillance between the Calgary Protocol (CP) and the recommended Canadian Urological Association Guidelines. Methods: A chart review using the Electronic Medical Record (Wolf) was assessed. 114 patients were on prostate cancer active surveillance. Biopsy results and intervals, complications, PSA intervals, PSA values and outcomes were extracted from all patient charts. All information was than collated with basic descriptive and bi-variable analyses were then performed.

Results: 114 patients were found to be on active surveillance with an average age of 61.3. Of those, 112 had prostate biopsies since October 2001. 53 had a repeat biopsy and of those 9 had a third biopsy. The mean time between the first and second biopsy was 16.5 months (3-37) and 27.6 months (18-56) between the second and third biopsy. CP deviated from CUA guidelines by 4.5 months and 10 months for the 1 st and second intervals, respectively. Repeat PSAs were taken at an average of 6.2 months (2-12.5). At the end of the study period, 96 patients were still on active surveillance, 2 were lost to follow up and 12 required treatment. Of the 12 patients who required intervention, 5 advanced from Gleason $3+3$ to $3+4$ upon repeat biopsy, and only one advanced from Gleason $3+3$ to $4+3$. Conclusion: Based on our histological grading of severity of disease, we feel time between biopsies averaging at 16.5 months between first and second biopsy and 27.6 months between second and third biopsy and combined with a PSA interval of 6.2 months was sufficient for early detection of progressive prostate cancer in our cohort.

Table 1. UP-56. Use of different surgical complication classification by journals

\begin{tabular}{lcccc}
\hline & EU & JU & BJUI & Uro \\
\hline C-D & 51 & 56 & 41 & 101 \\
Descriptive & 51 & 139 & 116 & 153 \\
CTCAE & 4 & 4 & 3 & 0 \\
ICS/IUGA & 0 & 1 & 0 & 0 \\
ICD-9/10 & 1 & 10 & 3 & 0 \\
Stephenson Classification & 1 & 0 & 1 & 1 \\
MSKCC & 1 & 0 & 1 & 0 \\
\hline EU: European Urology; JU: Journal of Urology; BJUI: BJU International; Uro: Urology; \\
C-D: Clavien-Dindo; CTCAE: Common Terminology Criteria for Adverse Events; ICS/ \\
IUGA: International Continence Society/lnternational Urogynecological Association; \\
ICD: International Classification of Diseases; MSKCC: Memorial Sloan-Kettering Cancer \\
Center.
\end{tabular}

\section{UP-56}

Use of Clavien-Dindo Classification in Reporting and Grading of Complications After Urologic Surgical Procedures: Analysis of 2010-2012

Yoon, Peter; Chalasani, Venu'; Woo ${ }^{1}$; Henry'; Winter, Matthew ${ }^{2}$

${ }^{1}$ The University of Sydney, Sydney, NSW, Australia; ${ }^{2}$ Urology Society Australia and New Zealand, Victoria, Australia

Introduction and Objectives: Report of postoperative complications is important measures for determining successful surgical outcome and quality. The absence of standardized guidelines for reporting surgical complications in the urological literature makes it difficult for readers to interpret the studies objectively. Recent EAU recommendations by Mitropoulos et al, compared the use of reporting systems between 1999-2000 and 2009-2010 showing a recent shift towards the use of standardized complication reporting such as the Clavien-Dindo Classification (CD) after urologic procedures and since this report, its use is anticipated to have further increased. This paper aims to analyze the use of CD in the Urological papers since 2010 through a systematic literature review and to assess the recent trend in use of different classifications by the authors for major urological journals. Methods: All articles from four major urological journals published between Jan 2010 and Oct 2012 were reviewed. All studies reporting surgical outcomes were included in the study and were individually assessed by retrieval of the full text. Case reports, reviews, meta-analyses, commentaries, and studies reporting a particular surgical complication or relatively non-invasive procedures were excluded. Use of different complication classifications were recorded with particular view on use of CD.

Results: Surgical outcomes were reported in 868 articles between Jan 2010 and Oct 2012 in four major urological journals. 'No complications' were reported in 130 articles. Use of different classification systems by journals is represented in Table 1 and Table 2. Use of CD Classification in all four journals increased from $20.96 \%$ in 2010 to $51.60 \%$ in 2012 .

Conclusions: The use of CD has been increasing over the years and is increasingly being adopted by the authors. Whilst use is increasing, there is still significant room for an improved uptake of standardized systems for reporting surgical complications.

Table 2. UP-56. Use of Clavien-Dindo classification by journals

\begin{tabular}{lccccc}
\hline & EU (\%) & JU (\%) & BJUI (\%) & Uro (\%) \\
\hline 2010 & $15 / 47(31.91)$ & $11 / 73(15.07)$ & $5 / 48(10.42)$ & $17 / 61(27.87)$ & Total (\%) \\
2011 & $17 / 34(50.00)$ & $22 / 78(28.02)$ & $17 / 68(25.00)$ & $32 / 110(29.09)$ & $88 / 229(20.96)$ \\
2012 & $19 / 28(67.86)$ & $23 / 59(38.98)$ & $19 / 48(39.58)$ & $52 / 84(61.90)$ & $113 / 219(51.60)$ \\
Total & $51 / 109(46.79)$ & $56 / 210(26.67)$ & $41 / 164(25.00)$ & $101 / 255(39.61)$ & $249 / 738(33.74)$ \\
\hline EU: European Urology; JU: Journal of Urology; BJUl: BJU International; Uro: Urology. & &
\end{tabular}

